\title{
Genetic and clinical characteristics of maturity-onset diabetes of the young in Chinese patients
}

Jian Yu Xu ${ }^{1}$, Qing Hong Dan ${ }^{1}$, Vivian Chan ${ }^{1}$, Nelson MS Wat ${ }^{1}$, Sidney Tam ${ }^{2}$, Sau Cheung $\mathrm{Tiu}^{3}$, Ka Fai Lee ${ }^{4}$, Shing Chung Siu ${ }^{5}$, Man Wo Tsang ${ }^{6}$, Lai Ming Fung ${ }^{7}$, Kin Wah Chan ${ }^{8}$ and Karen SL Lam*,1

${ }^{1}$ Department of Medicine, University of Hong Kong, Queen Mary Hospital, Hong Kong, China; ${ }^{2}$ Clinical Biochemistry Unit, University of Hong Kong, Queen Mary Hospital, Hong Kong, China; ${ }^{3}$ Department of Medicine, Queen Elizabeth Hospital, Hong Kong, China; ${ }^{4}$ Department of Medicine, Kwong Wah Hospital, Hong Kong, China; ${ }^{5}$ Department of Medicine, Tung Wah Eastern Hospital, Hong Kong, China; ${ }^{6}$ Department of Medicine, United Christian Hospital, Hong Kong, China; ${ }^{7}$ Department of Medicine, Caritas Medical Centre, Hong Kong, China; ${ }^{8}$ Department of Medicine, Princess Margaret Hospital, Hong Kong, China

In Caucasians, maturity-onset diabetes of the young (MODY) is mostly caused by mutations in the hepatocyte nuclear factor (HNF)-1 $\alpha$ (MODY3) and glucokinase (MODY2) genes. Most Japanese MODY patients, however, are not linked to known MODY genes. In this study, we examined the genetic and clinical characteristics of Chinese subjects with MODY. The study included 146 unrelated families fulfilling the minimum criteria for MODY: two consecutive generations of type II diabetes with at least one member diagnosed under the age of 25. We screened for mutations in the HNF-4 $\alpha$ (MODY1), MODY2 and MODY3 genes by direct sequencing. Antibody to glutamic acid decarboxylase (GAD-Ab) was measured in subjects with MODY of unknown cause (MODYX). Insulin resistance index and other clinical data were compared in sex-, age- and duration-matched MODY 3 and MODYX patients. In all, 13 families had MODY 3 mutations and two had MODY2 mutations. No MODY1 mutation was found. Four of the 12 different MODY 3 mutations were newly identified novel mutations (Q243E, A311D, P379R and P488fsdelC). In subjects with MODYX, $3 \%$ were GAD-Ab positive and $60 \%$ were overweight. Compared to MODY3 patients, MODYX patients had higher body mass index $(P<0.02)$, higher insulin resistance index $(P=0.001)$ and triglyceride level $(P<0.02)$, lower HDL level $(P=0.001)$ and more hypertension $(P<0.05)$, but no significant difference in the prevalence of diabetic complications. In conclusion, MODY 3 and MODY2 account for only 9 and $1 \%$, respectively, of Chinese MODY. A majority of Chinese MODY patients are due to defects in unknown genes and appear to be characterized by insulin resistance.

European Journal of Human Genetics (2005) 13, 422-427. doi:10.1038/sj.ejhg.5201347

Published online 15 December 2004

Keywords: maturity-onset diabetes of the young; obesity; insulin resistance; Chinese

${ }^{*}$ Correspondence: Professor KSL Lam, Division of Endocrinology, University Department of Medicine, University of Hong Kong, Queen Mary Hospital, Hong Kong, China. Tel: + 868522855 4769; Fax: +86 8522816 2187; E-mail: ksllam@hkucc.hku.hk

Received 3 May 2004; revised 29 July 2004; accepted 30 July 2004
Introduction

Type II diabetes mellitus (T2DM) refers to a diverse group of metabolic diseases, which are characterized by both impaired $\beta$-cell function and insulin resistance. In Hong Kong, its prevalence is reaching alarming dimensions (9.8\% among adults). ${ }^{1}$ Studies involving over 350000 persons have shown that diabetic patients have significant 
reductions in life expectancy. ${ }^{2}$ Furthermore, the younger an individual is diagnosed with diabetes, the larger reductions in life expectancy he or she will suffer. Knowledge of the etiology of T2DM will help in the primary prevention of diabetes and its complications, which are important public health priorities. However, despite extensive research in the recent decade, the major genes causing common T2DM remain elusive.

Maturity-onset diabetes of the young (MODY) is a monogenic type of T2DM, characterized by an early age of onset (usually at $\leqslant 25$ years of age) and an autosomal dominant mode of inheritance. To date, six genes have been identified to be responsible for MODY. These include the hepatocyte nuclear factor $(H N F)-4 \alpha$ gene (MODY1), ${ }^{3}$ glucokinase (GCK) gene (MODY2), ${ }^{4} H N F-1 \alpha$ gene $(\mathrm{MODY} 3){ }^{5}$ insulin promoter factor-1 (IPF-1) gene (MODY4), ${ }^{6} \mathrm{HNF}-1 \beta$ gene (MODY5) ${ }^{7}$ and neurogenic differentiation factor 1 (NEUROD1) gene (MODY6). ${ }^{8}$ The identification of these MODY genes has made considerable contribution to our understanding of the pathogenesis of common T2DM. MODY3 and MODY2 are the two most prevalent forms, accounting for more than $80 \%$ of MODY patients in Caucasians. ${ }^{9}$ Mutations in the MODY1 gene are less common, and are found in one out of 57 in Japanese subjects with MODY. ${ }^{10}$ Other subtypes are rare disorders reported only in a few families. ${ }^{6-8}$ Previous studies suggest that about $80 \%$ of Japanese MODY patients cannot be explained by known MODY genes. ${ }^{11}$ Hence, there is a need for more extensive genetic studies on Asian patients with MODY.

In Hong Kong Chinese, MODY3 and MODY2 mutations were found to be responsible for 5 and 4\%, respectively, of familial T2DM presenting before the age of $40 .{ }^{12}$ Our previous study on 50 MODY families revealed the prevalence of MODY3 to be $12 \% .{ }^{13}$ However, due to the small number of MODY families included, the actual prevalence of Chinese MODY subtypes and their clinical characteristics remain to be ascertained. In this study, we performed the screening for mutations in the MODY1, MODY2 and MODY3 genes in 146 unrelated clinical MODY families; we also characterized the phenotypes of MODY3 (the most commonly identified cause of MODY) and MODY with unknown cause (MODYX).

\section{Subjects and methods}

\section{Subjects and clinical studies}

Building upon our previous study of 50 Chinese MODY families, ${ }^{13}$ the current study collected an additional 96 Chinese MODY families from seven diabetes centers in Hong Kong, bringing our combined sample up to 146 families. All these families fulfilled the minimal criteria for MODY: two consecutive generations of type II diabetes with at least one member diagnosed at $\leqslant 25$ years of age. ${ }^{9}$ Of them, there were 41 families with diabetes in three consecutive generations. A total of 481 individuals were evaluated, including 365 affected subjects. The 146 probands were the first patients presented to us in each family and diagnosed with $\mathrm{T} 2 \mathrm{DM}$ at $\leqslant 25$ years. The mean age at diagnosis of these probands was $20.37 \pm 3.40$ years. The other 219 affected subjects included 179 parents, 29 siblings and 11 other relatives. A total of 100 unrelated Chinese subjects (aged 63.23 \pm 4.98 years) with normal glucose tolerance as determined by the results of a 75-g oral glucose tolerance test and no family history of diabetes were selected as controls from a previous cohort study. ${ }^{14}$ All subjects gave informed consent and the protocol was approved by the Ethics Committee, The University of Hong Kong.

The following clinical data were obtained from each subject: age, duration of diabetes, treatment, body mass index (BMI), waist hip ratio (WHR), blood pressure, fasting plasma glucose (FPG), glycosylated hemoglobin $\left(\mathrm{HbA}_{1} \mathrm{c}\right)$, lipid profile (total, LDL and HDL cholesterol and triglyceride), serum alanine aminotransferase (ALT), albumin, creatinine and evidence of diabetic microvascular and macrovascular complications, assessed as described previously. ${ }^{14,15}$ A blood sample was also taken for DNA extraction and determinations of antibody to glutamic acid decarboxylase (GAD-Ab) by enzyme-linked immunosorbent assay (Roche Diagnostics, Mannheim, Germany) and fasting C-peptide by radioimmunoassay (DiaSorin Corporation Inc., MN, USA). Subjects with BMI $\geqslant 25 \mathrm{~kg} /$ $\mathrm{m}^{2}$ were considered as overweight. Since over $25 \%$ of the patients were on insulin, insulin resistance was assessed by an index modified from the homeostasis model assessment for insulin resistance index $\left(\mathrm{HOMA}_{\mathrm{IR}}=\mathrm{FPG} \times\right.$ fasting insulin/22.5), using instead the product of FPG and fasting Cpeptide. ${ }^{16,17}$

\section{Screening for mutations in the MODY1, MODY2 and MODY3 genes}

The exons, flanking introns and minimal promoter of the MODY1, MODY2 and MODY3 genes were amplified by polymerase chain reaction (PCR) as described previously. ${ }^{12,13}$ Bi-directional sequencing was performed using an ABI PRISM dRhodamine Dye Terminator Cycle Sequencing Kit on an ABI Prism 377 DNA Sequencer (Applied Biosystems, Foster City, CA, USA). The occurrence of putative mutations in control subjects was determined by restriction enzyme digestion if the nucleotide substitution affected an enzyme recognition site or, if not, by direct sequencing.

\section{Statistics}

Statistical analysis was performed using Statistical Package for Social Sciences (SPSS for Windows, version 10.0). For quantitative traits, Student's $t$-test was used. For qualitative traits, $\chi^{2}$ test or Fisher's exact test was used as appropriate. Difference of insulin resistance index between MODY3 and 
MODYX groups was also tested with analysis of covariance with $\mathrm{HbA}_{1 \mathrm{c}}$ as the covariate. $P<0.05$ was considered statistically significant.

\section{Results Identification of mutations}

In addition to the six previously reported MODY3 families, ${ }^{13}$ we also found two MODY2 families in the first 50 MODY families analyzed. No MODY1 family was detected. In view of the expected low frequency of MODY1 mutations, the other 96 MODY families were only screened for mutations in the MODY2 and MODY3 genes. Seven MODY3 families, but no MODY2 families, were found in the expanded MODY cohort. The mutations identified from screening of 146 families are listed in Table 1. MODY3 mutations Q243E, A311D, P379R and P488fsdelC were novel and had not been reported previously.

Out of the 15 MODY3 or MODY2 families, nine had family members recruited for cosegregation study (Figure 1). Cosegregation of a mutation with diabetes was seen in MODY3 families \#37, \#113, \#41, \#212, \#217, \#236 and \#249 (Figure 1a, c, d and f-i). In these seven families, mutations were detected in the probands and all the other 17 affected family members with DNA available, including seven parents, seven siblings, two grandparents and one aunt. None of the 12 nondiabetic family members carried the mutations. Segregation was inconclusive in Family \#52 and \#194 (Figure 1b and e). In these two families, several diabetic relatives did not carry the mutations. For the other six families, Family \#9, \#26, \#97, \#101, \#235 and \#238, DNA from family members with diabetes could not be obtained for study.

\section{Clinical characteristics of Chinese MODY3 and MODYX subjects}

One MODY3 family (\#194) and 45 of 131 MODYX families had bilineal inheritance of T2DM. In the 13 MODY3 families, there were 36 diabetic subjects with DNA available, and 33 out of the 36 (92\%) diabetic subjects were MODY3 mutation carriers. The BMI of 33 diabetic MODY3 mutation carriers was $22.22 \pm 3.31 \mathrm{~kg} / \mathrm{m}^{2}$. In all, 21 of these subjects including the 13 probands were diagnosed under the age of 25 ; the age of diagnosis of the remaining 12 subjects ranged from 30 to 67 years. The BMI of the 131 MODYX probands was $26.35 \pm 4.77 \mathrm{~kg} / \mathrm{m}^{2}$, with $60 \%$ of them being overweight. To evaluate the contribution of autoimmunity to the pathogenesis of MODYX, GAD-Ab was measured and four (3\%) were found to be positive.

To better define the phenotypic characteristics of Chinese MODY3 and MODYX, we compared 21 MODY3 mutation carriers with the presentation of diabetes at $\leqslant 25$ years from 13 families with 63 sex-, age- and durationmatched MODYX probands who were GAD-Ab negative (Table 2). MODYX subjects had higher BMI, higher prevalence of hypertension and, being overweight, higher triglyceride and lower HDL levels than MODY3 subjects. Moreover, MODYX subjects had higher levels of FPG, $\mathrm{HbA}_{1 \mathrm{c}}$, fasting $\mathrm{C}$-peptide and insulin resistance index. The difference in insulin resistance index remained significant after being adjusted for $\mathrm{HbA}_{1 \mathrm{c}}(P<0.001)$. No difference was observed in the levels of serum albumin, ALT and creatinine, or the prevalence of diabetic complications. There was no significant difference in the percentage of patients requiring insulin or oral hypoglycemic agents (OHA), although fewer patients with MODYX could be controlled with diet alone.

Table 1 Mutations in the MODY3 and MODY2 genes

\begin{tabular}{|c|c|c|c|c|c|}
\hline \multirow[b]{2}{*}{ Family } & \multicolumn{2}{|c|}{ Location of mutation } & \multirow[b]{2}{*}{ Nucleotide change } & \multirow[b]{2}{*}{ Amino-acid change } & \multirow[b]{2}{*}{ Designation } \\
\hline & Exon/intron & Codon/nucleotide & & & \\
\hline \multicolumn{6}{|l|}{ MODY3 } \\
\hline$\# 9^{a}$ & 2 & 176 & $\mathrm{CAG} \rightarrow \mathrm{TAG}$ & Gln $\rightarrow$ Stop & Q176X \\
\hline$\# 26^{a}, \# 249$ & 2 & 171 & $\mathrm{CGA} \rightarrow \mathrm{TGA}$ & Arg $\rightarrow$ Stop & $\mathrm{R} 171 \mathrm{X}$ \\
\hline$\# 37^{a^{\prime}}$ & 6 & 379 & Deletion of $\mathrm{CT}$ & Frameshift & P379fsdelCT \\
\hline$\# 41^{\mathrm{a}}$ & 1 & 20 & $\mathrm{GGG} \rightarrow \mathrm{AGG}$ & Gly $\rightarrow$ Arg & G20R \\
\hline$\# 101^{\mathrm{a}}$ & 2 & 112 & $\mathrm{CCG} \rightarrow \mathrm{CTG}$ & Pro $\rightarrow$ Leu & P112L \\
\hline$\# 113^{\mathrm{a}}$ & Intron7 & nt-6 & $\mathrm{G} \rightarrow \mathrm{A}$ & - & IVS7nt-6 G $\rightarrow$ A \\
\hline \#194 & 4 & 311 & $\mathrm{GCC} \rightarrow \mathrm{GAC}$ & $\mathrm{Ala} \rightarrow \mathrm{Asp}$ & $\mathrm{A} 311 \mathrm{D}^{\mathrm{b}}$ \\
\hline \#212 & 6 & 379 & $\mathrm{CCT} \rightarrow \mathrm{CGT}$ & Pro $\rightarrow$ Arg & P379R \\
\hline \#217 & 4 & 243 & $\mathrm{CAG} \rightarrow \mathrm{GAG}$ & $\mathrm{Gln} \rightarrow \mathrm{Glu}$ & Q243E ${ }^{b}$ \\
\hline \#235 & Intron5 & $\mathrm{nt}+2$ & $\mathrm{~T} \rightarrow \mathrm{A}$ & - & IVS5nt $+2 T \rightarrow A$ \\
\hline \#236 & 7 & 488 & Deletion of C & Frameshift & P488fsdel $C^{b}$ \\
\hline \#238 & 3 & 203 & $\mathrm{CGT} \rightarrow \mathrm{CAT}$ & $\mathrm{Arg} \rightarrow \mathrm{His}$ & $\mathrm{R} 203 \mathrm{H}$ \\
\hline MODY2 & & & & & \\
\hline \#52, \#97 & 7 & 239 & $\mathrm{CAG} \rightarrow \mathrm{CGG}$ & Gln $\rightarrow$ Arg & Q239R \\
\hline
\end{tabular}

${ }^{\mathrm{a}}$ Families reported in previous publication. ${ }^{13}$

${ }^{\mathrm{b}}$ Newly identified novel mutations, and not detected in 100 controls. Mutation found in intron is noted with respect to the splice acceptor site (-) or splice donor site $(+)$. nt: nucleotide. 
\#37

P379fsdelCT HNF-1 $\alpha$

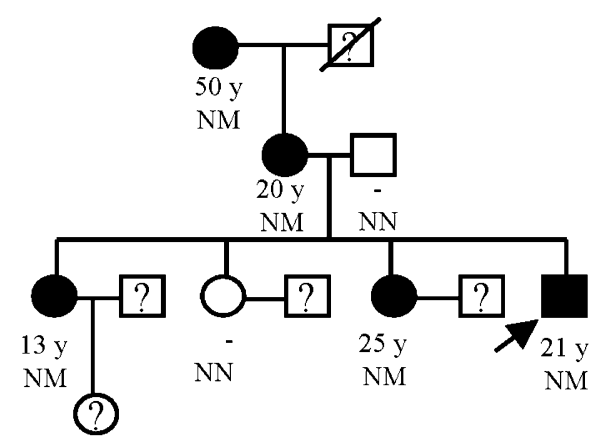

d

\#41

G20R $H N F-1 \alpha$

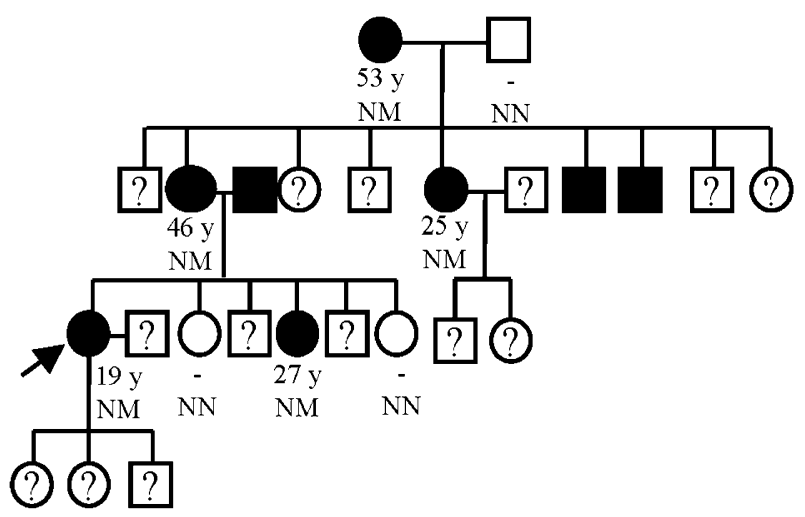

g h

\#236

P488fsdelC $H N F-I \alpha$

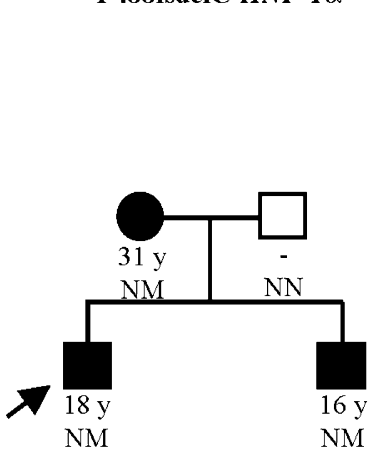

b

$\# 52$

Q239R GCK

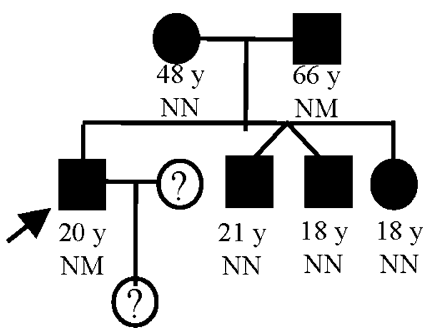

e

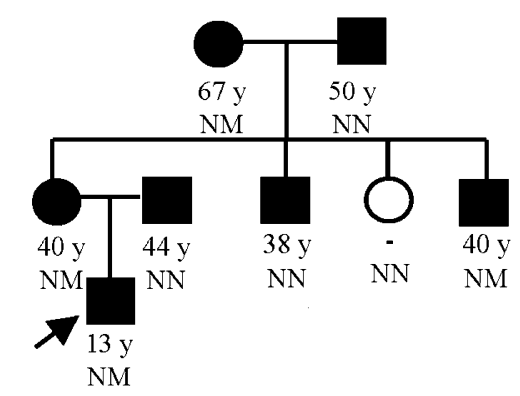

C \#113

IVS7-6 G $\rightarrow \mathrm{A} H N F-1 \alpha$

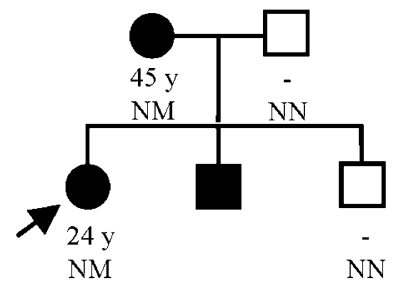

f

\#212

P379R HNF-1 $\alpha$

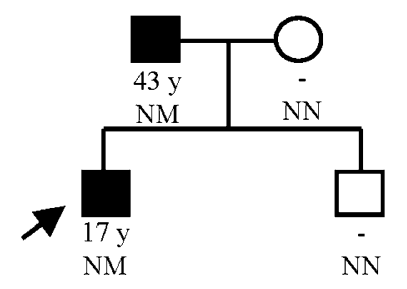

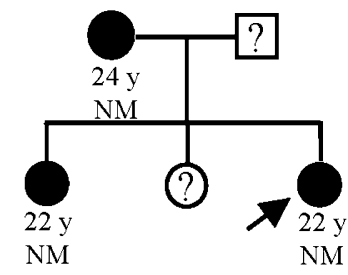

i

\#249

R171X $H N F-1 \alpha$

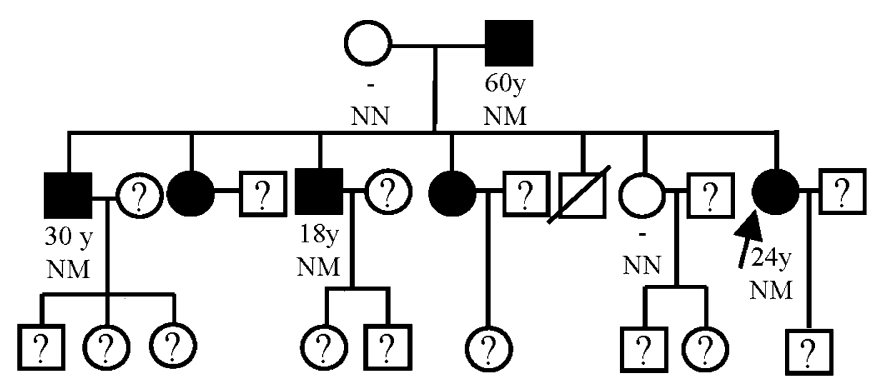

Figure 1 Pedigrees of families with MODY3 and MODY2 mutations. $\bullet$, diabetic female; $\mathbf{\square}$, diabetic male; $\bigcirc \square$, subjects with normal glucose tolerance; 10 , untested subjects; $\nearrow$, Proband. Age at diagnosis (years) and genotype, if known, are indicated. $\mathrm{N}$, normal; $\mathrm{M}$, mutation.

\section{Discussion}

In this study, the prevalence of MODY3 and MODY2 was only 9 and 1\%, respectively, in 146 unrelated Chinese MODY families. Among the MODY3 mutations, G20R, P112L, R171X, Q176X and P379fsdelCT have been shown to impair or cause a loss in HNF-1 $\alpha$ transactivation activity. ${ }^{13}$ The intronic mutation IVS7nt- $6 \mathrm{G} \rightarrow \mathrm{A}$ has been reported to cause abnormal splicing of $H N F-1 \alpha .{ }^{18}$ The other intronic mutation IVS5nt $+2 \mathrm{~T} \rightarrow \mathrm{A}$ is at a splice donor site and would affect splicing. The frameshift mutation P488fsdelC generates a truncated protein, which loses about $30 \%$ of transactivation domain. The missense mutations R203H, Q243E, A311D and P379R are predicted to change the protein structure, according to the Chou- 
Table 2 Clinical characteristics of sex-, age- and duration-matched MODY3 and MODYX subjects

\begin{tabular}{|c|c|c|c|}
\hline & MODY3 & MODYX & P-value \\
\hline Number (male/female) & $21(9 / 12)$ & $63(24 / 39)$ & 0.699 \\
\hline Age (years) & $32.14 \pm 10.12$ & $31.68 \pm 9.33$ & 0.848 \\
\hline Age at diagnosis (years) & $21.24+4.71$ & $20.95+3.13$ & 0.797 \\
\hline Duration of diabetes (years) & $10.95 \pm 9.06$ & $10.71 \pm 8.44$ & 0.913 \\
\hline BMI $\left(\mathrm{kg} / \mathrm{m}^{2}\right)$ & $22.59 \pm 3.45$ & $25.43 \pm 4.63$ & 0.012 \\
\hline Overweight ( $\mathrm{n}$ and \%) & $5(24 \%)$ & $36(56 \%)$ & 0.008 \\
\hline WHR & $0.84 \pm 0.06$ & $0.88 \pm 0.07$ & 0.066 \\
\hline $\mathrm{FPG}(\mathrm{mmol} / \mathrm{l})$ & $6.7(5.4-10)$ & $8.1(6.4-10.6)$ & $0.005^{\mathrm{a}}$ \\
\hline $\mathrm{HbA}_{1 \mathrm{c}}(\%)$ & $7.47 \pm 1.17$ & $8.20 \pm 1.95$ & 0.045 \\
\hline Triglyceride ( $\mathrm{mmol} / \mathrm{l})$ & $0.81(0.63-1.2)$ & $1.5(0 . \overline{9}-2.13)$ & $0.016^{\mathrm{a}}$ \\
\hline Cholesterol $(\mathrm{mmol} / \mathrm{l})$ & $4.72 \pm 0.79$ & $4.87 \pm 1.00$ & 0.538 \\
\hline LDL cholesterol (mmol/l) & $2.84 \pm 0.62$ & $3.02 \pm 0.89$ & 0.405 \\
\hline HDL cholesterol (mmol/l) & $1.4(1.10-1.63)$ & $1.1(0.95-1.2)$ & $0.001^{\mathrm{a}}$ \\
\hline Serum creatinine $(\mu \mathrm{mmol} / \mathrm{l})$ & $76(67.5-91)$ & $74(65-86)$ & $0.607^{\mathrm{a}}$ \\
\hline $\operatorname{ALT}(I U / I)$ & $22(17.5-37)$ & $20(14-31)$ & $0.179^{\mathrm{a}}$ \\
\hline Serum albumin $(\mathrm{g} / \mathrm{l})$ & $43(41-44.5)$ & $43(40-45)$ & $0.200^{\mathrm{a}}$ \\
\hline Fasting C-peptide (nmol/l) & $0.26(0.20-0.48)$ & $0.46(0.34-0.71)$ & $<0.001^{\mathrm{a}}$ \\
\hline FPGC-peptide & $1.74(1.16-2.87)$ & $3.89(2.39-6.27)$ & $0.001^{\mathrm{a}}$ \\
\hline Hypertension ( $n$ and \%) & $1(5 \%)$ & $18(29 \%)$ & 0.033 \\
\hline Albuminuria ( $n$ and \%) & $1(4.7 \%)$ & $7(11 \%)$ & 0.673 \\
\hline Retinopathy ( $n$ and \%) & $4(19 \%)$ & $15(24 \%)$ & 0.770 \\
\hline Neuropathy ( $n$ and \%) & $1(5 \%)$ & $4(6 \%)$ & $>0.999$ \\
\hline Macrovascular complication ( $n$ and \%) & 0 & $2(3 \%)$ & $>0.999$ \\
\hline \multicolumn{4}{|l|}{ Treatment } \\
\hline Insulin ( $n$ and \%) & $5(24 \%)$ & $21(33.33 \%)$ & 0.414 \\
\hline $\mathrm{OHA}(n$ and \%) & $11(52 \%)$ & $38(60.32 \%)$ & 0.523 \\
\hline Diet only ( $n$ and \%) & $5(24 \%)$ & $4(6.35 \%)$ & 0.039 \\
\hline
\end{tabular}

For continuous variables, normally distributed data were expressed as the mean $\pm \mathrm{SD}$, and data with skewed distribution were expressed as median (interquartile range).

aValues were logarithmically transformed for statistics.

Fasman plot. ${ }^{19}$ The MODY2 mutation Q239R, which has been previously reported, ${ }^{12}$ changes a residue that is conserved in human, rat and mouse. The non-cosegregation of A311D and Q239R with diabetes could not exclude their diabetogenic role, because the two families had bilineal inheritance of T2DM (Figure $1 \mathrm{~b}$ and e). In these two families, diabetes in the members who did not carry the mutation A311D or Q239R might have been caused by an unknown diabetic gene inherited from the diabetic parent without the respective MODY gene mutation. None of the mutations was detected in the 100 nondiabetic controls and, based on the family cosegregation studies, reported functional studies or anticipated changes in amino acids, all mutations are likely to have functional significance.

We have not screened for mutations in the MODY4, MODY5 or MODY6 genes. These subtypes are rare, and have been excluded in Japanese MODY families. ${ }^{20,21}$ Furthermore, MODY5 is associated with renal dysfunction due to defects in kidney development, which were not present in our MODY patients. Therefore, these subtypes of MODY are unlikely to contribute to diabetes in a significant proportion of our MODY families, and about $90 \%$ of our Chinese MODY families are not linked to known
MODY genes. However, since 45 of the 131 MODYX families have bilineal transmission of diabetes, it is possible that these MODYX families, despite fitting the minimal MODY criteria, may be early-onset polygenic T2DM that, through chance, has resulted in an apparently autosomal dominant pattern of inheritance.

It is interesting to note that only 21 of the 33 MODY3 mutation carriers had diagnosis of diabetes at $\leqslant 25$ years of age. The other 12 MODY3 mutation carriers, despite the presence of a MODY3 mutation, were actually diagnosed at a later age, ranging from 30 to 67 years, and would be considered clinically as early-onset or even late-onset type II diabetes.

During the past decade, an increasing frequency in the occurrence of T2DM has been reported in adolescents, and this increase seems to parallel the increase in prevalence of obesity. The majority of our MODYX subjects were overweight, suggesting an important role of excess adiposity in the pathogenesis of MODYX in our population, as in common T2DM. As only 3\% of these MODYX subjects were GAD-antibody positive, autoimmunity is apparently not a major underlying factor of MODYX in Chinese.

MODY3 subjects had similar liver and kidney function, and frequency of diabetic complications, as age- and 
duration-matched MODYX subjects. These results were in agreement with those reported in other populations. ${ }^{22,23}$ On the other hand, over half of the MODYX subjects were overweight, compared to only $24 \%$ in the MODY3 subjects. Furthermore, the higher BMI and triglyceride, lower HDL and a higher prevalence of hypertension in MODYX subjects suggested the presence of greater insulin resistance compared to the MODY3 subjects. This was confirmed by the presence of a higher insulin resistance index that was independent of the difference in glycemic control. Taken together, these data suggest that the insulin resistance syndrome forms a major part of our MODYX phenotype. This was also observed in Caucasian subjects with earlyonset T2DM unlinked to known MODY genes. ${ }^{24}$

In conclusion, consistent with studies in Japanese, ${ }^{11}$ this study suggests that MODYX accounts for $80-90 \%$ of clinical MODY cases in Asians. Thus, we propose that, in contrast to findings in Caucasians, ${ }^{9}$ the major gene for MODY in Asians remains to be identified. We have shown that excess adiposity and insulin resistance are common features of Chinese patients with MODYX. The elucidation of the molecular genetics of MODYX is likely to result in better understanding of the pathogenesis of obesity, insulin resistance and common T2DM.

\section{Acknowledgements}

We thank the staff in the Diabetic Centers of QMH, QEH, KWH, TWE, UCH, CMC and PMH for their efforts in recruiting the families, and Ms Kok-Weng Chan for technical assistance. This study was supported by a CRCG grant from the University of Hong Kong and a seeding grant from the Department of Medicine, University of Hong Kong.

\section{References}

1 Janus ED, Wat NM, Lam KSL et al: The prevalence of diabetes, association with cardiovascular risk factors and implications of diagnostic criteria (ADA 1997 and WHO 1998) in a 1996 community-based population study in Hong Kong Chinese. Hong Kong Cardiovascular Risk Factor Steering Committee. Am Diabet Assoc Diabet Med 2000; 17: 741-745.

2 Narayan KM, Boyle JP, Thompson TJ, Sorensen SW, Williamson DF: Lifetime risk for diabetes mellitus in the United States. JAMA 2003; 290: 1884-1890.

3 Yamagata K, Furuta $\mathrm{H}$, Oda $\mathrm{N}$ et al: Mutations in the hepatocyte nuclear factor 4 alpha gene in maturity-onset diabetes of the young (MODY1). Nature 1996; 384: 458-460.

4 Stoffel M, Froguel PH, Takeda J et al: Human glucokinase gene: isolation, characterization, and identification of two missense mutations linked to early-onset non-insulin-dependent (type 2) diabetes mellitus. Proc Natl Acad Sci USA 1992; 89: 7698-7702.

5 Yamagata K, Oda N, Kaisaki PJ et al: Mutations in the hepatic nuclear factor 1 alpha gene in maturity-onset diabetes of the young (MODY3). Nature 1996; 384: 455-458.
6 Stoffers DA, Ferrer J, Clarke WL, Habener JF: Early-onset type-II diabetes mellitus (MODY4) linked to IPF1. Nat Genet 1997; 17: $138-139$.

7 Horikawa Y, Iwasaki N, Hara M et al: Mutation in hepatocyte nuclear factor-1 $\beta$ gene (TCF2) associated with MODY. Nat Genet 1997; 17: 384-385.

8 Malecki MT, Jhala US, Antonellis A et al: Mutations in NEUROD1 are associated with the development of type 2 diabetes mellitus. Nat Genet 1999; 23: 323-328.

9 Frayling TM, Evans JC, Bulman MP et al: Molecular and clinical characterization of mutations in transcription factors. Diabetes 2001; 50 (Suppl 1): S94-S100.

10 Furuta $\mathrm{H}$, Iwasaki $\mathrm{N}$, Oda $\mathrm{N}$ et al: Organization and partial sequence of the hepatocyte nuclear factor- $4 \alpha / \mathrm{MODY} 1$ gene and identification of a missense mutation, R127W, in a Japanese family with MODY. Diabetes 1997; 46: 1652-1657.

11 Yamada S, Zhu Q, Aihara Y et al: Cloning of cDNA and the gene encoding human hepatocyte nuclear factor (HNF)- $3 \beta$ and mutation screening in Japanese subjects with maturity-onset diabetes of the young. Diabetologia 2000; 43: 121-124.

$12 \mathrm{Ng}$ MCY, Lee SC, Ko GTC et al: Familial early-onset type 2 diabetes in Chinese patients. Diabetes Care 2001; 24: 663-671.

$13 \mathrm{Xu}$ JY, Chan V, Zhang WY, Wat NM, Lam KSL: Mutations in the hepatocyte nuclear factor-1alpha gene in Chinese MODY families: prevalence and functional analysis. Diabetologia 2002; 45: $744-746$.

14 Wat NMS, Lam TH, Janus ED, Lam KSL: Central obesity predicts the worsening of glycaemia in Southern Chinese. Int J Obes Relat Metab Disord 2001; 25: 1789-1793.

15 Lam KSL, Pang RWC, Wat MS, Lauder IJ, Janus ED: Apolipoprotein (a) levels and phenotypes in NIDDM patients with microalbuminuria and albuminuria. Nephrol Dial Transplant 1996; 11: $2229-2236$.

16 Matthews DR, Hosker JP, Rudenski AS, Naylor BA, Treacher DF, Turner RC: Homeostasis model assessment (HOMA): insulin resistance and $\beta$-cell function from fasting plasma glucose and insulin concentrations in man. Diabetologia 1985; 28: 412-419.

17 Gutt M, Davis CL, Spitzer SB et al: Validation of the insulin sensitivity index (ISI $(0,120))$ : comparison with other measures. Diabetes Res Clin Pract 2000; 47: 177-184.

18 Bulman MP, Harries LW, Hansen T et al: Abnormal splicing of hepatocyte nuclear factor 1 alpha in maturity-onset diabetes of the young. Diabetologia 2002; 45: 1463-1467.

19 Chou PY, Fasman GD: Prediction of the secondary structure of proteins from their amino acid sequences. Adv Enzymol 1978; 47: $45-148$.

20 Hara M, Lindner TH, Paz VP et al: Mutations in the coding region of the insulin promoter factor 1 gene are not a common cause of maturity-onset diabetes of the young in Japanese subjects. Diabetes 1998; 47: 845-846.

21 Furuta $\mathrm{H}$, Horikawa $\mathrm{Y}$, Iwasaki $\mathrm{N}$ et al: Mutations in the coding region of the BETA2/NeuroD1 (NEUROD1) and Nkx2.2 (NKX2B) genes are not associated with maturity-onset diabetes of the young in Japanese. Diabetes 1998; 47: 1356-1358.

22 Isomaa $\mathrm{B}$, Henricsson $\mathrm{M}$, Lehto $\mathrm{M}$ et al: Chronic diabetic complications in patients with MODY3 diabetes. Diabetologia 1998; 41: 467-473.

23 Iwasaki $\mathrm{N}$, Ogata $\mathrm{M}$, Tomonaga $\mathrm{O}$ et al: Liver and kidney function in Japanese patients with maturity-onset diabetes of the young. Diabetes Care 1998; 21: 2144-2148.

24 Doria A, Yang Y, Malecki M et al: Phenotypic characteristics of early-onset autosomal-dominant type 2 diabetes unlinked to known maturity-onset diabetes of the young (MODY) genes. Diabetes Care 1999; 22: 253-261. 